\title{
No influence of FAT polymorphisms in response to aripiprazole
}

\author{
Chi-Un Pae ${ }^{1,2}$, Alberto Chiesa ${ }^{3}$, Laura Mandelli ${ }^{3}$, Diana De Ronchi ${ }^{3}$ and Alessandro Serretti ${ }^{3}$
}

The aim of this study was to investigate possible influences of a set of markers in the FAT gene (rs2306987, rs2306990, rs2637777 and rs2304865) on efficacy and tolerability of aripiprazole in the treatment of schizophrenic patients. Efficacy was assessed at baseline and weeks 1, 2, 4, 6, 8 and 12 using the Clinical Global Impression Severity and Improvement scale (CGI-S; CGI-I), the Brief Psychiatric Rating Scale and the Schedule for the Assessment of Negative Symptoms scale. Side effects were evaluated by means of the Simpson-Angus Scale for Extrapyramidal Symptoms, the Barnes Akathisia Scale and the Abnormal Involuntary Movement Scale. Multivariate analyses were employed to test possible influences of single nucleotide polymorphisms on clinical and safety variables. Analysis of haplotypes was also performed. No relevant association between FAT variants and clinical or safety scores was observed. Haplotype analysis did not reveal any significant association with clinical and safety scores at any time as well. Our data suggest no association between investigated alleles and genotypes in $F A T$ and response to aripiprazole. However, because several limitations characterize the present study, further investigations on larger studies are required.

Journal of Human Genetics (2010) 55, 32-36; doi:10.1038/jhg.2009.117; published online 6 November 2009

Keywords: aripiprazole; cadherins; pharmacogenetics; schizophrenia

\section{INTRODUCTION}

The cadherins constitute a superfamily of cell-surface glycoproteins, many of which participate in cell-cell adhesion and recognition. ${ }^{1}$ The founding members of the superfamily, commonly described as classical (or type 1) cadherins, occur in most solid tissues of the body. ${ }^{2}$ Furthermore they guide neuronal migration and positioning during development, processes that are believed to participate to the development of schizophrenia and bipolar disorder.,

Growing attention has recently been given to a gene that is closely related to the classical cadherin genes family. This gene encodes a transmembrane protein containing 34 cadherin repeats in association with a number of other motifs (tumor suppressor 1 precursor or $F A T){ }^{5}$ The $F A T$ gene is located in chromosome $4 \mathrm{q} 35.2$ and it spans about $136 \mathrm{~kb}$. The atypical cadherin FAT exerts its effect as a receptor for a signaling pathway that regulates growth, gene expression and planar cell polarity. Although current studies mainly focused on the role of classical cadherins and FAT in cancer, ${ }^{5,6}$ there is consistent evidence suggesting a possible role of FAT in the development of psychiatric disorders. $^{7-11}$

Blair et al. ${ }^{8}$ recently reported FAT as a susceptibility gene for bipolar disorder. They found that a haplotype block at the $3^{\prime}$ end of the cadherin gene FAT (rs2306987, rs1298865, rs2306990, rs2637777 and rs2304865) associated to bipolar disorder in an Australian patientcontrol sample of 137 families. More recently, Abou Jamra et al. ${ }^{7}$ replicated and extended previous findings observing that some single nucleotide polymorphisms (SNPs) in FAT (rs4862718, rs7683023, rs2306987, rs1298865, rs2306990, rs10009030, rs2637777, rs2304865 and rs328437) were significantly associated with bipolar disorder in an independent sample of German subjects. However, negative results have been also reported. ${ }^{8}$

Increasing evidence suggests that bipolar disorder and schizophrenia could share many risk genes, ${ }^{12-14}$ although evidence for cadherins is still limited. ${ }^{4}$ Note, however, that the role of FAT in neuronal migration and positioning during development makes it a good candidate gene for schizophrenia. To date, however, no study has yet focused on a possible role of FAT on the response to psychotropic treatments. In a first attempt to extend findings observed in bipolar patients to schizophrenia patients, we preliminarily investigated the influence of a set of SNPs in FAT gene, chosen on the basis of previous findings (rs2306987, rs2306990, rs2637777 and rs2304865), on clinical and safety outcomes in a sample of Korean patients suffering from schizophrenia and treated with aripiprazole.

\section{MATERIALS AND METHODS}

Sample description

Detailed sample description has been reported in a previous paper. ${ }^{15}$ Briefly, out of 177 screened schizophrenia inpatients or outpatients according to Diagnostic and Statistical Manual of Mental Disorders, fourth edition criteria,

${ }^{1}$ Department of Psychiatry, Seoul St Mary's Hospital, The Catholic University of Korea College of Medicine, Seoul, Republic of Korea; ${ }^{2}$ Department of Psychiatry, Holy Family Hospital, The Catholic University of Korea College of Medicine, Kyounggi-Do, Republic of Korea and 3Institute of Psychiatry 'P Ottonello', University of Bologna, Bologna, Italy Correspondence: Professor A Serretti, Department of Psychiatry, University of Bologna, Viale Carlo Pepoli 5, Bologna 40123, Italy.

E-mail: alessandro.serretti@unibo.it

Received 11 September 2009; accepted 14 October 2009; published online 6 November 2009 
87 were included into the intent-to-treat sample. Patients were eligible for inclusion if their symptoms were not optimally controlled and/or if they did not tolerate current antipsychotic medications well. Patients had been randomly assigned to three different switching conditions to aripiprazole $(10 \mathrm{mg})$ by (1) simultaneous discontinuation of current antipsychotic, (2) gradual tapering off current antipsychotic over a period of 4 weeks with half dose after the first 2 weeks or (3) gradual tapering off current antipsychotic over 4 weeks after maintenance of current dose for 2 weeks. Patients were then followed for 12 weeks with the original aim to assess efficacy and tolerability of different switching strategies. Rescue benzodiazepines (less than $4 \mathrm{mg}$ per day of diazepam or equivalent) and antiparkinsonian medication were allowed in case of acute extrapyramidal symptoms, but not for prophylactic use. No significant difference in the number of benzodiazepines' users and dosage administered was found among the three groups. ${ }^{15}$ The study protocol, including genetic analysis, was approved by the institutional review board of Kangnam St Mary's Hospital.

\section{Efficacy and safety evaluations}

Efficacy was assessed using the Clinical Global Impression Severity and Improvement Scale (CGI-S; CGI-I). ${ }^{16}$ Other measures of outcome employed were the Brief Psychiatric Rating Scale (BPRS $)^{17}$ and the Schedule for the Assessment of Negative Symptoms (SANS). ${ }^{18}$ Side effects were evaluated by the Simpson-Angus Scale for Extrapyramidal Symptoms (SAS),${ }^{19}$ the Barnes Akathisia Scale $(\mathrm{BAS})^{20}$ and the Abnormal Involuntary Movement Scale (AIMS). ${ }^{21}$ All evaluators were blind to the group allocation of patients. There was good reliability among evaluators $(k>0.8)$.

\section{DNA analysis}

Genomic DNA was extracted from blood by standard methods and quantified. The high-throughput genotyping method using pyrosequencer (Biotage $\mathrm{AB}$, Uppsala, Sweden) was used for genotyping four SNPs (rs2306987, rs2306990, rs2637777 and rs2304865) within the FAT gene. PCR primers (Bioneer, Daejeon, Korea) and sequencing primers (Bioneer) used for the pyrosequencing assay were designed by using the Pyrosequencing Assay Design Software version 1 (Biotage $\mathrm{AB}$ ) and one primer of each primer set was biotinylated. A map of the positions of the four SNPs under investigation in FAT gene is shown in Figure 1.

\section{Statistical analysis}

Statistical analyses were performed using the Statistica package (StatSoft Italia srl, Vigonza (PD), Italy). ${ }^{22}$ Multivariate analysis of covariance (MANCOVA) was used to test the influence of SNPs on efficacy and safety indexes. To reduce possible sources of bias, we included sex and age as covariates in the MANCOVA model. Haploview 3.2 (Daly Lab, Broad Institute, Cambridge, MA, USA) was used to generate a linkage disequilibrium (LD) map and to test for Hardy-Weinberg equilibrium (HWE) ${ }^{23}$ Tests for associations using multimarker haplotypes were performed using the statistics environment ' $\mathrm{R}$ ' (http:// www.R-project.org), package 'haplo.score', to compare clinical and safety scores at each evaluation's point among haplotypes. Sex, age and switching strategy were added as covariates. Permutations $(n=10000)$ were performed to estimate the global significance of the results for all haplotypes analyses and to validate the expectation-maximization values.

All $P$-values were two-tailed, and, in accordance with Bonferroni correction for multiple testing (seven tests), statistical significance was conservatively set at the 0.007 to reduce the likelihood of false-positive results. With these parameters, we had a sufficient power $(0.80)$ to detect a large effect size of 0.78 , allowing to detect, for example, a difference of 0.87 points on the difference of week 1 CGI-S between men and women and corresponding to a variance explained of $13.7 \% .^{24}$

\section{RESULTS}

Socio-demographic features of the sample

Demographic and baseline clinical features are reported in Table 1. There were some significant associations between genotypes and clinical-demographic and safety scores at baseline. In the following analysis, we thus systematically controlled for them to reduce possible sources of variance. As previously observed, ${ }^{15}$ severity scores significantly improved during the study whereas no significant difference was observed for side effects.

\section{Hardy-Weinberg equilibrium and linkage disequilibrium for DTNBP1}

All markers were in HWE: rs2306987(A/T), $P=0.625$; rs2306990(T/C), $P=1.0 ;$ rs2637777(G/T), $P=0.64$; rs2304865(G/C), $P=0.81$. Strong LD was observed in the whole block $(0.88$ between rs2306987 and rs2637777; 0.93 between rs2306990 and rs2637777; 0.89 between rs2637777 and rs2304865; and 1 among the remaining SNPs). Genotypes and alleles prevalence were consistent with frequencies observed in Asian populations according to international databases (http://snpper.chip.org/).

\section{Allele, genotype and haplotype analysis}

No significant association was found between rs2306987, rs2306990, rs2637777 and rs2304865 alleles or genotypes and clinical or safety assessments at any time of observation or over time as measured by repeated-measures ANCOVA (all $P$-values $>0.007$ ). Haplotype analysis did not reveal any significant association with clinical and safety scores as well (all $P$-values $>0.007$ ).

\section{DISCUSSION}

In this study, we preliminarily investigated the potential influence of a set of markers in the FAT gene on clinical outcome and risk for side effects associated to aripiprazole treatment in a sample of
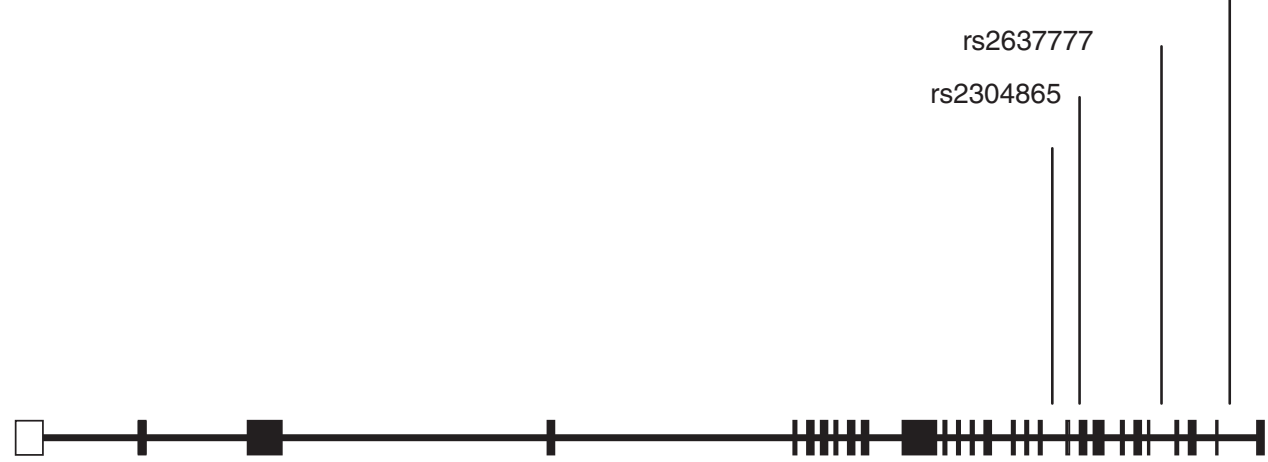

Figure 1 FAT gene and polymorphisms under investigation. 
Table 1 FAT SNPs considered in this study and baseline characteristics

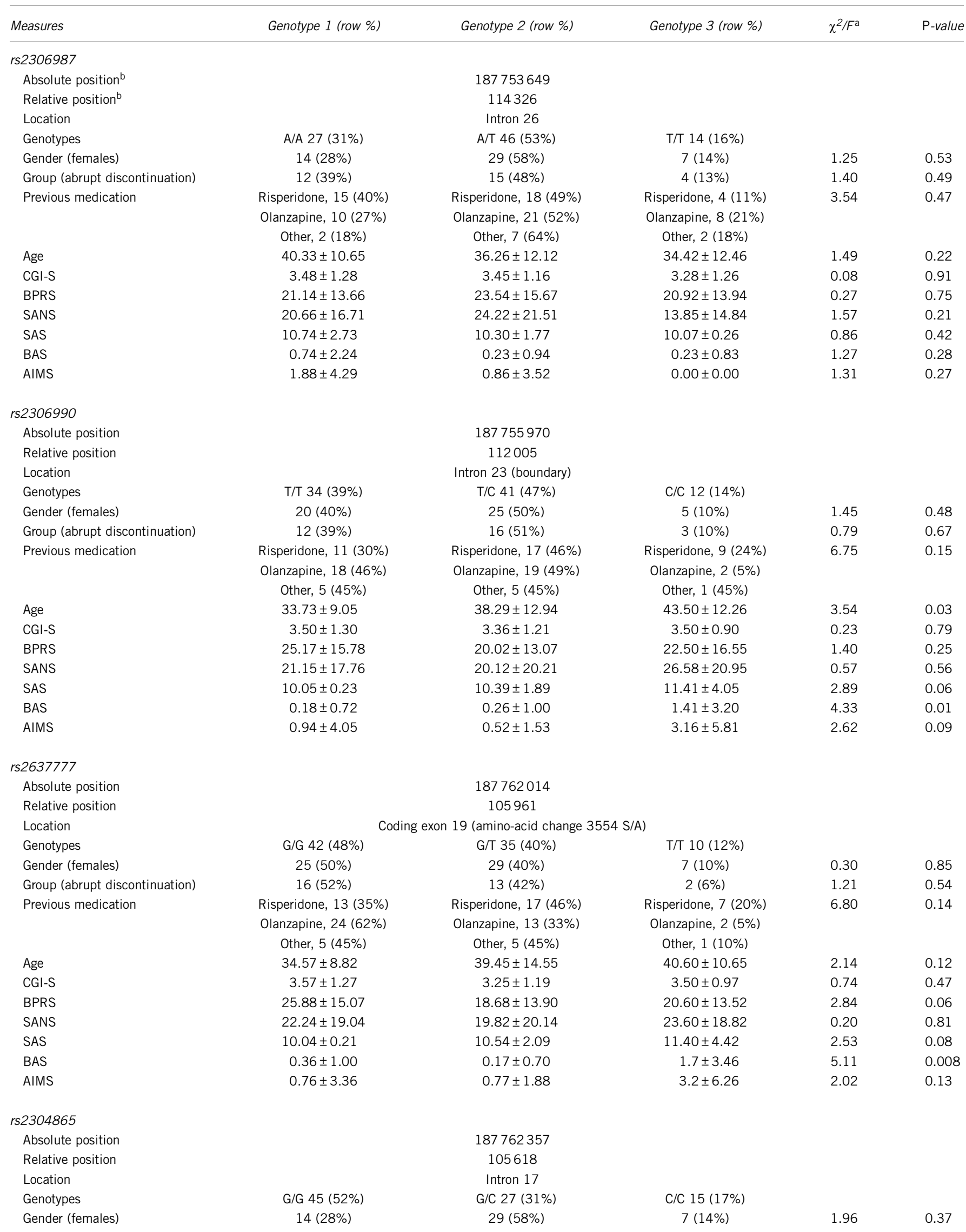


Table 1 (Continued)

\begin{tabular}{|c|c|c|c|c|c|}
\hline Measures & Genotype 1 (row \%) & Genotype 2 (row \%) & Genotype 3 (row \%) & $\chi^{2} / F^{a}$ & $\mathrm{P}$-value \\
\hline Group (abrupt discontinuation) & $12(39 \%)$ & $14(45 \%)$ & $5(16 \%)$ & 1.34 & 0.50 \\
\hline \multirow[t]{3}{*}{ Previous medication } & Risperidone, 15 (40\%) & Risperidone, 18 (49\%) & Risperidone, 4 (11\%) & 4.06 & 0.39 \\
\hline & Olanzapine, 10 (26\%) & Olanzapine, 20 (51\%) & Olanzapine, 9 (23\%) & & \\
\hline & Other, 7 (64\%) & Other, 2 (18\%) & Other, $2(18 \%)$ & & \\
\hline Age & $40.33 \pm 10.65$ & $36.42 \pm 12.21$ & $34.06 \pm 12.09$ & 1.59 & 0.21 \\
\hline CGI-S & $3.48 \pm 1.28$ & $3.44 \pm 1.17$ & $3.33 \pm 1.23$ & 0.04 & 0.95 \\
\hline BPRS & $21.14 \pm 13.66$ & $22.86 \pm 15.15$ & $23.13 \pm 15.92$ & 0.19 & 0.82 \\
\hline SANS & $20.66 \pm 16.71$ & $24.29 \pm 21.76$ & $14.33 \pm 14.42$ & 1.54 & 0.21 \\
\hline SAS & $10.74 \pm 2.73$ & $10.31 \pm 1.79$ & $10.66 \pm 0.25$ & 0.88 & 0.41 \\
\hline BAS & $0.74 \pm 2.24$ & $0.24 \pm 0.95$ & $0.21 \pm 0.80$ & 1.27 & 0.28 \\
\hline AIMS & $1.88 \pm 4.29$ & $0.88 \pm 3.56$ & $0.00 \pm 0.00$ & 1.33 & 0.26 \\
\hline
\end{tabular}

Global sample

Total number of subjects

Gender (females)

Group (abrupt discontinuation)

Previous medication

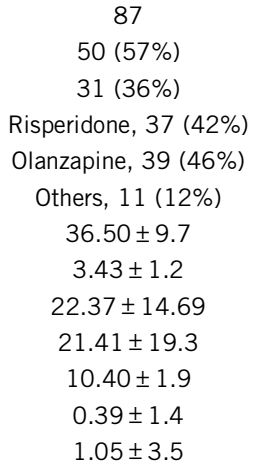

Abbreviations: AIMS, Assessment of Involuntary Movement Scale; BAS, Barnes Akathisia Scale; BPRS, Brief Psychiatric Rating Scale; CGI-S, Clinical Global Impression Severity; SANS, Schedule for the Assessment of Negative Symptoms; SAS, Simpson-Angus Scale.

aThe $\chi^{2}$ statistic is referred to gender, group and previous medication. The $\mathrm{F}$ statistic is referred to other variables.

bAll data from snpper.chip.org. The relative position is related to the starting codon.

schizophrenia patients who were partial responders to a previous antipsychotic treatment. No significant association between alleles, genotypes or haplotypes under investigation and clinical or safety scores at any time or over time was observed, hence suggesting that FAT gene does not have a role in the response to aripiprazole in schizophrenia patients.

Note, however, that our findings should be considered with caution. Previous works observed a possible link between FAT gene and bipolar disorder ${ }^{7,8}$ and some have suggested an involvement of FAT in schizophrenia, ${ }^{3,4}$. However, no work has yet confirmed neither an association between FAT gene and response to moodstabilizing agents nor an association between FAT and schizophrenia. As a consequence, further studies are needed to investigate such possible associations. For this reason, our work should be considered as preliminary in that its significance could be better estimated if associations mentioned above were already confirmed.

In addition, because of several methodological limitations, our results cannot be generalized and deserve further investigations. First, given that we investigated only the switch to aripiprazole, it cannot be ruled out that the SNPs under investigation could influence the response to other antipsychotic agents, as well as to other psychotropic treatments. A further limitation of this study is represented by the small size of our sample, which could have limited the possibility to detect smaller effects of genetic variations on outcome scores. Indeed, we had no sufficient power to identify small effects, as these are usually exerted by single genes.
Ethnicity should be also considered, because susceptible and protective haplotypes could vary according to ethnic differences. ${ }^{25,26}$ Thus, we cannot rule out a possible association between FAT gene and response to aripiprazole in non-Korean samples. Further, we did not investigate a number of factors potentially influencing the course of symptoms over time independently from treatment, such as psychosocial status or environmental stressors. Also, the choice of SNPs was based on previous research ${ }^{7,8}$ and focused only on the $3^{\prime}$ of FAT gene; for this reason, further research is needed to provide a more complete coverage of FAT gene and to investigate further variants that could have a role on the expression of the gene and/or functionality of the protein.

Taking into account such restrictions, there is a preliminary indication of no influence of FAT gene on response to aripiprazole treatment over the short term. However, further research is required to confirm our finding in larger samples in order to better establish the role of $F A T$ on the efficacy and tolerability of aripiprazole and other antipsychotic treatments in other populations of patients.

\section{ACKNOWLEDGEMENTS}

This work was supported by a grant from the Medical Research Center, Korea Science and Engineering Foundation, Republic of Korea (R13-2002-005-04001-0). The founding source had no other role in the preparation of the paper.

1 Goodwin, M. \& Yap, A. S. Classical cadherin adhesion molecules: coordinating cell adhesion, signaling and the cytoskeleton. J. Mol. Histol. 35, 839-844 (2004). 
2 Tepass, U., Truong, K., Godt, D., Ikura, M. \& Peifer, M. Cadherins in embryonic and neural morphogenesis. Nat. Rev. Mol. Cell. Biol. 1, 91-100 (2000).

3 Yagi, T. \& Takeichi, M. Cadherin superfamily genes: functions, genomic organization, and neurologic diversity. Genes Dev. 14, 1169-1180 (2000).

4 Pedrosa, E., Stefanescu, R., Margolis, B., Petruolo, O., Lo, Y., Nolan, K. et al. Analysis of protocadherin alpha gene enhancer polymorphism in bipolar disorder and schizophrenia. Schizophr. Res. 102, 210-219 (2008).

5 Mahoney, P. A., Weber, U., Onofrechuk, P., Biessmann, H., Bryant, P. J. \& Goodman, C. $\mathrm{S}$. The fat tumor suppressor gene in Drosophila encodes a novel member of the cadherin gene superfamily. Cell 67, 853-868 (1991).

6 Jeanes, A., Gottardi, C. J. \& Yap, A. S. Cadherins and cancer: how does cadherin dysfunction promote tumor progression? Oncogene 27, 6920-6929 (2008).

7 Abou Jamra, R., Becker, T., Georgi, A., Feulner, T., Schumacher, J., Stromaier, J. et al. Genetic variation of the FAT gene at $4 \mathrm{q} 35$ is associated with bipolar affective disorder. Mol. Psychiatry 13, 277-284 (2008).

8 Blair, I. P., Chetcuti, A. F., Badenhop, R. F., Scimone, A., Moses, M. J., Adams, L. J. et al. Positional cloning, association analysis and expression studies provide convergent evidence that the cadherin gene FAT contains a bipolar disorder susceptibility allele. Mol. Psychiatry 11, 372-383 (2006).

9 Friddle, C., Koskela, R., Ranade, K., Hebert, J., Cargill, M., Clark, C. D. et al. Fullgenome scan for linkage in 50 families segregating the bipolar affective disease phenotype. Am. J. Hum. Genet. 66, 205-215 (2000).

10 McInnis, M. G., Lan, T. H., Willour, V. L., McMahon, F. J., Simpson, S. G., Addington, A. M. et al. Genome-wide scan of bipolar disorder in 65 pedigrees: supportive evidence for linkage at $8 \mathrm{q} 24,18 \mathrm{q} 22,4 \mathrm{q} 32,2 \mathrm{p} 12$, and 13q12. Mol. Psychiatry 8, 288-298 (2003).

11 Willour, V. L., Zandi, P. P., Huo, Y., Diggs, T. L., Chellis, J. L., MacKinnon, D. F. et al. Genome scan of the fifty-six bipolar pedigrees from the NIMH genetics initiative replication sample: chromosomes 4, 7, 9, 18, 19, 20, and 21. Am. J. Med. Genet. B Neuropsychiatr. Genet. 121, 21-27 (2003).

12 Porteous, D. Genetic causality in schizophrenia and bipolar disorder: out with the old and in with the new. Curr. Opin. Genet. Dev. 18, 229-234 (2008).
13 Ivleva, E., Thaker, G. \& Tamminga, C. A. Comparing genes and phenomenology in the major psychoses: schizophrenia and bipolar 1 disorder. Schizophr. Bull. 34, 734-742 (2008).

14 Craddock, N., O'Donovan, M. C. \& Owen, M. J. Genes for schizophrenia and bipolar disorder? Implications for psychiatric nosology. Schizophr. Bull. 32, 9-16 (2006).

15 Pae, C. U., Serretti, A., Chiesa, A., Mandelli, L., Lee, C., Kim, J. et al. Immediate versus gradual suspension of previous treatments during switch to aripiprazole: results of a randomized, open label study. Eur. Neuropsychopharmacol. 19, 562-570 (2009)

16 Guy, W. Clinical Global Impression. Assessment Manual for Psychopharmacology 217-222 (Department of Health Education and Welfare, Washington, DC, USA, 1976).

17 Flemenbaum, A. \& Zimmermann, R. L. Inter- and intra-rater reliability of the Brief Psychiatric Rating Scale. Psychol. Rep. 32, 783-792 (1973).

18 Andreasen, N. C. The Scale for the Assessment of Negative Symptoms (SANS): conceptual and theoretical foundations. Br. J. Psychiatry Suppl. 7, 49-58 (1989).

19 Simpson, G. M. \& Angus, J. W. A rating scale for extrapyramidal side effects. Acta. Psychiatr. Scand. Suppl. 212, 11-19 (1970).

20 Barnes, T. R. A rating scale for drug-induced akathisia. Br. J. Psychiatry 154, 672-676 (1989).

21 Branch, P. R. Abnormal Involuntary Movement Scale (AIMS). Early Clinical Drug Evaluation Unit Intercom. 4, 3-6 (1975)

22 StatSoft I: STATISTICA per Windows, StatSoft Italia srl, Vigonza (PD) (1995).

23 Barrett, J. C., Fry, B., Maller, J. \& Daly, M. J. Haploview: analysis and visualization of LD and haplotype maps. Bioinformatics 21, 263-265 (2005).

24 Cohen, J. Statistical Power Analysis for the Behavioral Sciences (Lawrence Erlbaum, Hillsdale, New Jersey, 1988).

25 Numakawa, T., Yagasaki, Y., Ishimoto, T., Okada, T., Suzuki, T., Iwata, N. et al. Evidence of novel neuronal functions of dysbindin, a susceptibility gene for schizophrenia. Hum. Mol. Genet. 13, 2699-2708 (2004).

26 Hwu, H. G., Lin, M. W., Lee, P. C., Lee, S. F., Ou-Yang, W. C. \& Liu, C. M. Evaluation of linkage of markers on chromosome $6 \mathrm{p}$ with schizophrenia in Taiwanese families. Am. J. Med. Genet. 96, 74-78 (2000). 